\title{
Research of Downhole Instructions Decoding with Variable Drilling Fluid Displacement
}

\author{
Aiqing HUO* \\ School of Electrical Engineering \\ Xi'an Shiyou University \\ Xi'an, China \\ E-mail: huoaiqing@sina.com \\ +* Corresponding author \\ Wei HE \\ School of Electrical Engineering \\ Xi'an Shiyou University \\ Xi'an, China \\ E-mail: 793569530@qq.com
}

\author{
Shaohui LI \\ School of Electrical Engineering \\ Xi'an Shiyou University \\ Xi'an, China \\ E-mail: 1059945352@qq.com
}

\author{
Ke SUN \\ School of Electrical Engineering \\ Xi'an Shiyou University \\ Xi'an, China \\ E-mail: 798372410@qq.com
}

\begin{abstract}
This paper studies a downlink communication mode in which the effective downhole control instruction was generated by the use of drilling fluid pump displacement to format three descending and three ascending pulse instruction encoding. The principle of the downlink communication was introduced. The encoding idea of the downlink instruction code with 5-bit pulse was described. This downlink code is used to compose the corresponding control instruction which includes the information of the tool face angle and the guide force percentage. The downhole pulse widths were recognized by using STM32 as a micro control chip. The corresponding control commands were identified through the development of the software in the downhole drilling tool. Laboratory ground experiments demonstrated the feasibility of this downlink transmission mode by using variable drilling fluid displacement and the achievability of instruction decoding software.
\end{abstract}

Keywords-drilling fluid displacemenet; downlink communication; STM32 chip; instruction decoding

\section{INTRODUCTION}

The rotary steering drilling tool is a sophisticated technique which involves many subjects and advanced technologies. It is called "drilling missile and guidance technology". Its core technique is mainly composed of ground monitoring system, downhole bias-oriented control and execution tool and two-way communication system[1-3]. Downlink communication is the key technology to realize the closed control of rotary steerable drilling system. Therefore, transmitting control command from the ground to the downhole is the most important research for achieving automatic closed directional drilling.

There have been numerous researches in this area both domestically and globally. Engelder proposed to monitor the rotation angle of drill string or rotation rate of drill string using tilt meter and three-axis magnetometer[4]. In the aspect of patent application, US7298285[5] and US2006225920[6], downhole signal reception is achieved by monitoring the rate changes in rotor, which is driven by a mud-powered engine in the downhole tool. Fu Xinsheng at Xi'an Shiyou University decoded the encoded signals, which were sent from the downhole, through the measurement of the vibrational signal at the drill bit[7]. Most of the issues are caused by the vulnerable output signals from the downhole sensors due to the restrictions from the geological formation parameters, or to the complexity of the physical structures[8]. Considering signal transmission mode and the factors of the communication rate, depth and reliability of the downlink communication, etc., the drilling fluid pulse transmission is selected as a downlink information transmission protocol[9]. As a turbo-motor-drive is built in as a part of the rotary drilling tool, there is no need to add additional sensors for downlink communication [10][11]. Thus combining these two ideas, a improvement principle was proposed by using of drilling fluid pump displacement to format three descending and three ascending pulse instruction encoding. Meanwhile, the encoding instructions on the ground were given and the instructions decoding software underground in the downhole drilling tool were designed in this paper..

\section{Basic Principle of Downlink Communication}

The signal transmission system with drilling fluid as the transmission medium on the ground is mainly made up of the ground monitoring system controlling the pulse valve, the drill string used as the transmission channel and the receiving device in the downhole electronic storehouse.

The ground transmission system changes the drilling fluid displacement in the drill string by controlling the switch of the pulse valve. When the pulse valve is opened, the displacement of the drilling fluid in the drill string is reduced to $20 \%$ of the full displacement, and the change of the displacement leads to the voltage and frequency of the downhole turbine motor is changed. The downhole hardware 
processing circuit and the decoding software recognize the pulse variation of the voltage and interpret it as a control instruction code. Thus the large closed-cycle control from the ground monitoring system to the downhole drilling tool system is realized.

To implement the collection of the output voltage of the turbo motor, it is necessary to amplify, detect, rectify, filter and A/D convert to the voltage output signal[12]. The MCU converts the acquired digital signal into the corresponding control instruction code, the specific circuit structure diagram shown in Figure 1. Stm32F103RCT6 chip is used as MCU in the Fig 1.

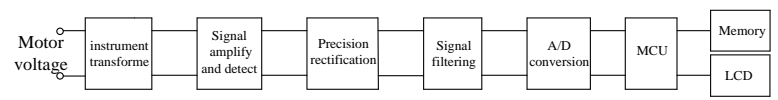

Figure 1. Structure diagram of downhole turbine motor voltage detection

\section{DOWNWARD INSTRUCTION ENCODING}

In order to realize the control of downhole drilling tools and to adjust the tool face angle and the guiding force percentage of the downhole drilling tools according to the expected requirements, the downlink instructions must be coded.

The three descending and three ascending encoding instructions[13] for drilling fluid displacement changed are shown in Fig 2. The receiving device can get the pulse width by the decoding software, pulse width is explained by five control information code bits, the first three bits represents the tool face angle, the fourth represents the guide force percentage, the last one is the parity bit.

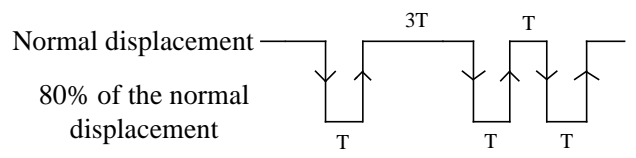

Figure 2. An encoding instruction

$\mathrm{T}$ is the minimum pulse width in the drilling fluid encoding instructions, which depends on the attenuation period of the drilling fluid fluctuation in the borehole, and the $\mathrm{T}$ is generally agreed to be 30s. Each pulse width is integer $m$ times than $\mathrm{T}$. In addition, in order to improve the sensitivity of the action of the downhole control mechanism and to ensure the accuracy of the test, it is stipulated that the total time of all the pulse widths of an effective instruction does not exceed $\mathrm{nT}$ (n is an integer), and each pulse width does not exceed $k T$, the biggest $\mathrm{k}$ is $9, \mathrm{k}<\mathrm{n}$ ). If the instruction recognized by the downhole receiving device meets the above conditions, it is regarded as a valid instruction.

\section{SOFTWARE DESIGN OF DOWNHOle INSTRUCTION DECODING}

Simple and efficient decoding software is need to design so as to decode the downward instruction accurately. In the scheme, the software programs perform the main tasks- the output voltage is collected by the driving $\mathrm{AD}$ chip, the voltage threshold range is set for pulse width, the control algorithm is gained from program codes, the LCD screen is drove to display the instruction codes and the instruction codes are stored in $\mathrm{I}^{2} \mathrm{C}$. Therefore, the contents about the pulse width identification, the count value reading, and the error instruction recognition will be presented in this section..

\section{A. Three-descending and Three-ascending Instruction Decoding}

The normal displacement and the pressure of drilling fluid are not a fixed value as different drilling technical parameters and casing dimensions. Thus, it is necessary to confirm a threshold voltage $\mathrm{V}$ of the turbine motor output corresponding to the full drilling fluid displacement, which is compared to the subsequent sample value Vi continually. The decoding software will be recognize it as a correct instruction when the change of two sampling values is more than $20 \% \mathrm{~V}$ meanwhile less than $60 \% \mathrm{~V}$. The threshold identification flow chart is shown in Fig 3.

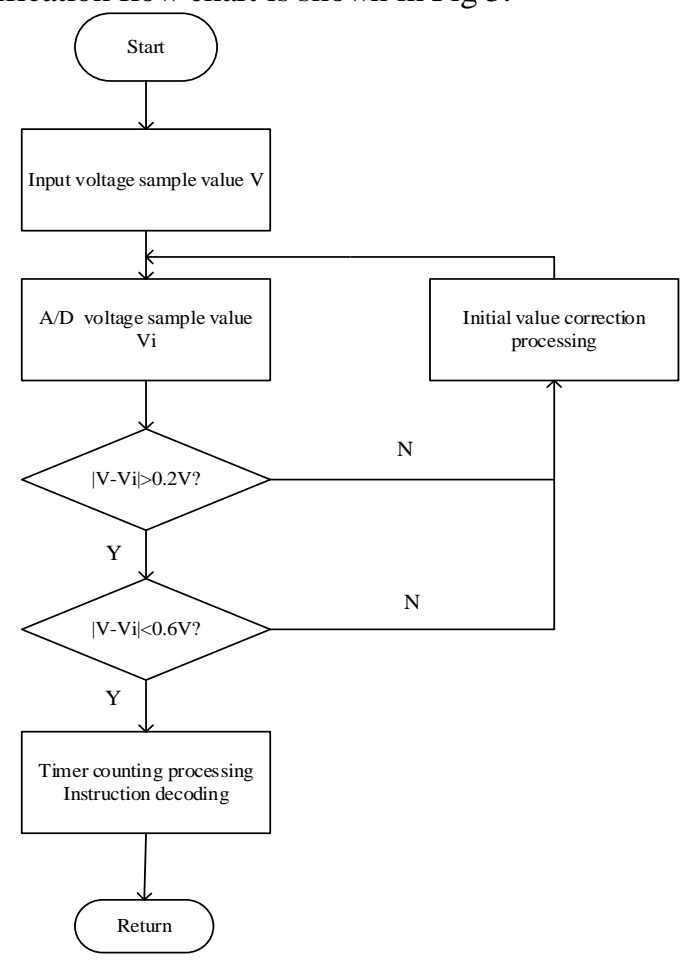

Figure 3. Threshold identification flow chart

The control instruction code is composed of corresponding number of 5-bit pulse width of threedescending and three-ascending, so the core of software design is to recognize the 5-bit pulse width. The software program compares the output voltage $\mathrm{Vi}$ from $\mathrm{A} / \mathrm{D}$ with the threshold voltage $\mathrm{V}$. The first descending edge arrives only when $\mathrm{Vi}=80 \% \mathrm{~V}( \pm 5 \%$ tolerance amplitude is allowed), that indicates the pulse output will be a low level. The idea of capturing low level pulse width is as follows: firstly, set TIM2_CH1 to capture the falling edge, waiting for the 
descending edge to capture the interruption. When the interruption of descending edge is captured, the sixth bit of the CAPTURE_STA register is will be set to 1 , which indicates that the low level is captured, then to start timer to count. Once the timer counter overflowing, the CAPTURE_STA register is used to count. At the same time, the TIM2_CH1 is set to capture the rising edge. The seventh bit of CAPTURE_STA is set to 1, which indicates that a low level is captured successfully. And the eighth bit of CAPTURE_STA is set to 1 by hardware. The flag is captured and the capture value of the timer is read to the CAPTURE_VAL register. Finally, the falling edge is set to return to the initial state. (Note: the timer is 16bits, the sampling frequency is set to $1 \mathrm{KHZ}$; CAPTURE_VAL register is 16 bits, maximum count is 65535; CAPTURE_STA register is 8 bits and the top six is overflow times for storage, the 7th is to capture low-level flag bit, the 8th is to capture complete flag bit). When $\mathrm{Vi}=$ $100 \% \mathrm{~V}$ (allows $\pm 5 \%$ deviation amplitude), it indicates that the first ascending edge arrives and pulse output is a high level. The start timer TIM5_CH1 captures the high pulse width. The capture process is not repeated as described above.

\section{B. Pulse Widths Value Obtaining}

For the timer to capture the count value, operation should be carried out by (1), where CAPTURE_STA \& OX3F is the number of overflows and CAPTURE_VAL is the capture value of the timer. Because the sampling frequency is $1 \mathrm{KHZ}$, the calculation time is milliseconds, divided by 1000 for seconds. The numerical value is converted into the corresponding instruction code by the minimum period $\mathrm{T}$, and the pulse width instruction is obtained. Since there is an error in this calculation, it is necessary to use the count value to find the minimum period. If the remainder is greater than $2 / 3$ of $T$, the command value is incremented by 1 , otherwise the command value will not change.

$$
t=\frac{(\text { CAPTURE_STA \& } 0 X 3 F) * 65535+C A P T U R E \_V A L}{1000}
$$

\section{Error Instruction Identification}

Due to the complex operating conditions in the underground, the signal is susceptible to interference in the transmission channel, resulting in abnormal drilling fluid pressure change, which lead to too narrow or too wide pulse width[14]. If the received pulse width time is $T<T$, the pulse width is too narrow; if the received pulse width time is $\mathrm{T}^{\prime}>$ 9T, the pulse width is too wide. All these cases are judged to be wrong instructions for processing. When receiving threedescending and three-ascending of voltage pulse signal, it need to start two timers, one is used to capture the normal pulse width, another one is to capture the interference pulse signal. If the interference signal time $\mathrm{T}^{\prime}$ is much smaller than the minimum period $\mathrm{T}$, it is considered as a peak interference. If the signal time $\mathrm{T}^{\prime}$ is more than 9T, it is considered as a wrong instruction which caused by the anomaly pressure in the hole.

After the instruction is successfully received, the algorithm is used to integrate the single instruction code into a 5-bit control instruction code. The control instruction is stored to 24C02 through $\mathrm{I}^{2} \mathrm{C}$ bus. Then the main CPU calls the stored control instruction to complete the control of the drilling tool, furthermore to realize the downlink communication.

\section{GROUND EXPERIMENT}

To simulate drilling fluid displacement changing, frequency converter is used to control the rotation of the drag motor in the ground simulation experiments. Moreover, the turbine motor is connected with the drag motor through the couple axle, so the change frequency of the converter is equal to the change of drilling fluid displacement, at the same time to change the output voltage of turbine motor. For example, when the frequency converter is $50 \mathrm{~Hz}$, it is equivalent to the normal pump displacement, and $40 \mathrm{~Hz}$ is equivalent to $80 \%$ of the pump displacement. The specific ground experimental platform is shown in Fig 4.

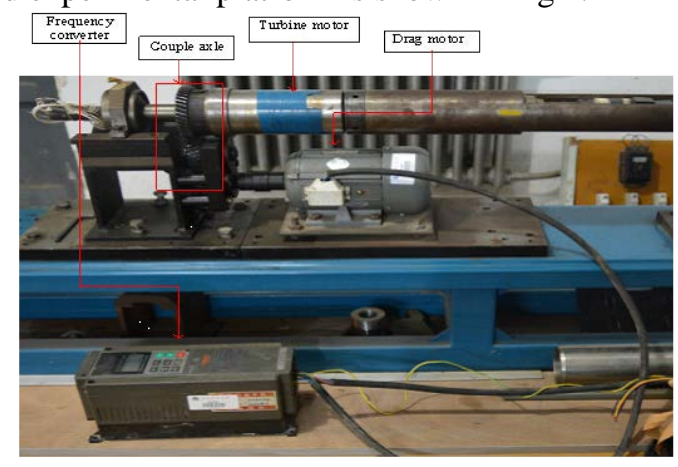

Figure 4. ground simulation experiment platform

A serie number of 13111 as an instruction code is sent on the ground simulation experiment platform, and the signal received by the turbine motor is shown in Fig 5 . The $\mathrm{AD}$ converter converts analog voltage into digital signal, which are interpreted by the instruction recognition program as the count of the pulse width to form the corresponding 5-bit control instruction code. The STM32 decode the pulse width consisting of 5-bit of instruction into 13111, where the first 3-bit in the instruction code 131 is represent the tool face angle $60^{\circ}$, the 4 th bit 1 is represent the steering force $100 \%$. The experimental results show that the software decoding can effectively identify the three descending and three ascending of 5-bit instruction code. 


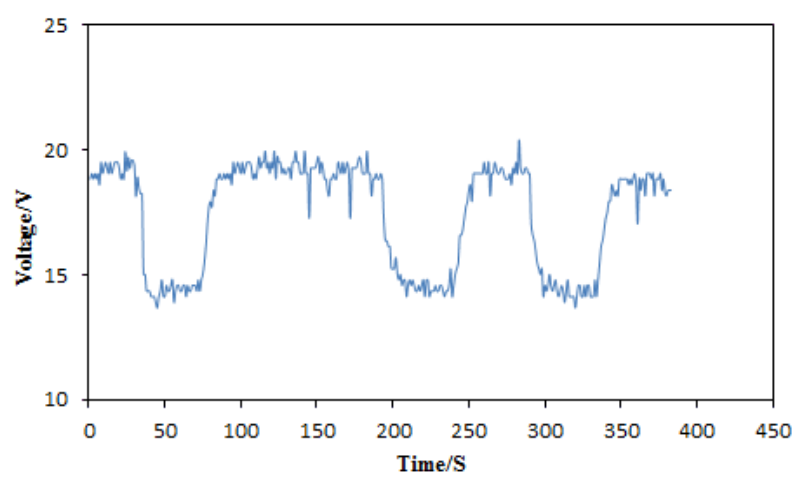

Figure 5. Downlink received instruction

\section{CONCLUSIONS}

1. It is a simple and attainable approach to use drilling fluid pulse with three-descending and three-ascending encoded instructions for downlink signal transmissions in a rotary steerable drilling system.

2. Falling edges and rising edges are captured by using two groups of timer and 5 pulses width are obtained by using a timer counting method, so that control instructions are identified in the downhole drilling tool.

3. Software programs are designed to judge and deal with error instructions encoding with spike disturbance, delay interference etc.

\section{ACKNOWLEDGMENT}

The authors greatly appreciate the supports from the colleagues at the Institute of Steerable Drilling of Xi'an Shiyou University. This paper is also supported by Shaanxi science and technology research and development program (2013 K07-16).

\section{REFERENCES}

[1] Wu Chao, Jian Zhi-jun ,Guo Yun, Shang Jie. Study on key techniques and drilling test of rotary steerable drilling system[J]. China offshore oil and gas.2012,24(3):52-53.
[2] Li feng-fei, Jiang Quan-shi, Li Han-xing, Gao De-li. Research on Signal Transmission System of Rotary Guided Drilling Tool[J].China's offshore oil and gas,2012,24(6):45-47.

[3] Tang Nan, HuoAi-qing, WangYue-long, Cheng Wei-bin. Development of downward communication receiving function in rotary steerable drilling system[J]. Journal of Petroleum Science, 2010,31 (1):157-160.

[4] Zong Yan-bo, Wang Lei, Ke Ke ,Sun Ming-guang .R \& D of downlink instruction recsivers for FPWD systems[J]. China offshore oil and gas. 2016.28(6):88-94.

[5] Zeng fang,Tang Bin ,Liang Ying,Zhang Xin. Research of haar lifting wavelet signal denoising on downward communication of steering drilling tool by drilling fliid displacement changes[J].Computer measurement and control.2014,22(6):1967-1969.

[6] Li San-wei, Shang Hai-yan,Zhou Jing.The rotatory directed command sends device controller research[J]. Information and communications, 2015.25(11):48-51.

[7] Tang Nan,Huo Ai-qing,Wang Yao-long.The rounding problem in rotary steerable drilling system downward signal solving[J].Computers and Applied Chemistry,2012,29(10):1167-1170.

[8] Huo Ai-qing, Dai Chen. Design of steering drilling downward communication ground monitoring system[J]. Xi 'an petroleum university journal , 2013.28(4):73-78.

[9] Huo Ai-qing, Tang Nan, Wang Yue-long, Cheng Wei-bin. Study of Downlink Instruction Transmission Receiver Based on Turbo Engine in Steerable Drilling[J]. Microcomputer Motor, 2013,41 (9):26-28.

[10] Huo Ai-qing,HE Yu-yao,WANG Yue-long,,TANG Nan, CHENG Wei-bin. Key Technology Research of Downward Communication Receiving System in Rotary Steerable Drilling.2010 2nd International Conference on Computer Engineering and Technology,April 1618,2010,chengdu,China,V1:pp413-416.

[11] Huo Ai-qing, HE Yu-yao, WANG Yue-long, TANG Nan, CHENG Wei-bin, Study of Downward Communication Receiver in Rotary Steerable Drilling Tool Based on Turbine Generator. Proceedings of the 29th Chinese Control Conference, July29-31, 2010, Beijing, China, pp4210-4213

[12] Wang Yue-long, Lv Jin-xing, Huo Ai-qing, Tang Nan. Accurate Measurement System for Downhole Turbine Electrical Machines[J].Microcomputer Motor,2010,11:24-26.

[13] Tang Nan, Wang Yue-long, Huo Ai-qing, Cheng Wei-bin.A downward signal processing method for rotary steerable drilling system based on signal similarity[J]. Petroleum exploration and development, 2012,39(1):111-114.

[14] Zhao Qi, Huo Ai-qing, Tang Nan. Design of Receiving Software of Downward Control Commands of Steering Drilling Tool Using Downhole Motor[J]. Computer Measurement and Control, 2010,18 (12):2898-2900. 